\title{
Diferencias en el salto vertical y la velocidad de patada mae-geri entre karatekas internacionales y nacionales
}

\author{
Carlos BALSALOBRE-FERNÁNDEZ ${ }^{*}$, Víctor MARTÍNEZ-MAJOLERO², Jorge VILLACIEROS- \\ RODRÍGUEZ ${ }^{3}$ \& Carlos Ma TEJERO-GONZÁLEZ1 \\ 1 Universidad Autónoma de Madrid. Departamento de Educación Física, Deporte y Motricidad Humana \\ (España) \\ 2 Colegio Santa Isabel de Madrid (España) \\ ${ }^{3}$ Federación Madrileña de Karate. Escuela Madrileña de Preparadores (España)
}

Recepción: 24/06/2013; Aceptación: 30/07/2013; Publicación: 01/08/2013.

ARTÍCULO ORIGINAL

\begin{abstract}
Resumen
Objetivos: La fuerza explosiva de miembros inferiores y la velocidad de la patada mae-geri son fundamentales en el karate de competición, aunque no está claro hasta qué punto estas variables diferencian a los competidores de mayor nivel deportivo. El objetivo de esta investigación es analizar las diferencias en la velocidad de ejecución de la patada maegeri y el salto vertical con contramovimiento (CMJ) entre un grupo de karatekas de máximo nivel internacional y otro grupo de karatekas de nivel nacional.

Método: Trece karatekas de nivel internacional y once karatekas de nivel nacional/amateur participaron en el estudio. Después de un calentamiento estándar, se midió la altura alcanzada (en $\mathrm{cm}$ ) en el CMJ mediante una plataforma de infrarrojos y la velocidad de ejecución (en $\mathrm{m} / \mathrm{s}$ ) de la patada mae-geri mediante cámaras de alta velocidad y análisis por software biomecánico.

Resultados: Procediendo mediante MANCOVA para analizar las diferencias entre los grupos controlando la variable edad, los resultados muestran que los karatekas internacionales tienen significativamente mayores niveles de CMJ que los nacionales $\left(+22.1 \%, \mathrm{~F}=9,47 ; p=0,006 ; \eta^{2}=0,311\right)$, no ocurriendo lo mismo con la velocidad de patada mae-geri $(+5,7 \%$, $\left.\mathrm{F}=0.80 ; p=0.38 ; \eta^{2}=0.03\right)$, que no mostró diferencias significativas entre grupos.

Conclusión: Nuestros datos muestran, por un lado, la importancia que tiene la valoración del CMJ como una herramienta para detectar talentos en karate y, por otro lado, que para alcanzar un nivel deportivo internacional en karate puede ser importante incrementar los niveles de CMJ hasta valores similares a los aquí ofrecidos.
\end{abstract}

Palabras clave: Artes marciales; deportes de combate; kata; fuerza explosiva; alto rendimiento.

Differences in vertical jumping and maegeri kicking velocity between international and national level karateka

\section{Abstract}

Aim: Lower limb explosive strength and mae-geri kicking velocity are fundamental in karate competition; although it is unclear whether these variables could differentiate the high-level athletes. The objective of this research is to analyze the differences in the mae-geri kicking velocity and the counter-movement jump (CMJ) between a group of international top level karateka and another group of national-level karateka.

Methods: Thirteen international-level karateka and eleven national-level karateka participated in the study. After a standard warm-up, CMJ height
Diferenças entre o salto vertical e a velocidade do pontapé mae-geri entre karatecas internacionais e nacionais

\section{Resumo}

Objetivos: A força explosiva dos membros inferiores e a velocidade do mae-geri são fundamentais no karaté de competição, pese embora não seja claro até que ponto estas variáveis se diferenciam nos competidores de maior nível desportivo. 0 objetivo desta investigação é analisar as diferenças de velocidade de execução do pontapé mae-geri e o salto vertical com contra-movimento (CMJ) entre um grupo de karatecas de alto nível internacional e um outro grupo de karatecas de nível nacional.

Método: Treze karatecas de nível internacional e onze karatecas de nível nacional/amadores participaram no estudo. Depois do normal (standard) aquecimento físico, mediu-se a altura alcançada $(\mathrm{em} \mathrm{cm})$ e o CMJ mediante uma plataforma de

* E-mail: carlos.balsalobre@uam.es. Dirección: Facultad de Formación de Profesorado y Educación. C/Francisco Tomás y Valiente, 3, Campus Cantoblanco Universidad Autónoma de Madrid. 28049. Madrid (España). 
(in $\mathrm{cm}$ ) and mae-geri kicking velocity (in $\mathrm{m} / \mathrm{s}$ ) was measured using an IR-platform and a highspeed camera, respectively.

Results: Proceeding with MANCOVA to analyze the differences between groups controlling the effect of age, the results show that the international-level karateka demonstrated significantly higher levels of $\mathrm{CMJ}$ than nationallevel competitors $\left(+22.1 \%, \mathrm{~F}=9.47, \mathrm{p}=0.006, \eta^{2}\right.$ $=0.311$ ). There were no significant differences between groups in the mae-geri kicking velocity $\left(+5,7 \%, \mathrm{~F}=0.80 ; p=0.38 ; \eta^{2}=0.03\right)$.

Conclusion: Our data shows, first, the importance of CMJ assessment as a tool to detect talent in karate and, second, that to achieve internationallevel in karate it may be important to increase $\mathrm{CMJ}$ levels to values similar to those offered here.

Keywords: Martial arts; combat sports; kata; explosive strength; high-performance. infravermelhos e a velocidade de execução (em m/s) do pontapé mae-geri mediante câmaras de alta velocidade e análise por software biomecânico.

Resultados: Procedimento mediante o teste estatístico MANCOVA para analisar as diferenças entre os grupos, controlando a variável idade, os resultados mostram que os karatecas internacionais têm significativamente maiores níveis de $\mathrm{CMJ}$ que os nacionais $(+22.1 \%, \mathrm{~F}=9,47 ; \mathrm{p}=0,006 ; \eta 2=0,311)$, não ocorrendo o mesmo com a velocidade do pontapé mae-geri $(+5,7 \%, F=0.80 ; p=0.38 ; \eta 2=0.03)$, que não mostrou diferenças significativas entre grupos.

Conclusão: Os nossos dados revelam, por um lado, a importância que tem a valorização do CMJ como uma ferramenta para detetar talentos no karaté e, por outro lado, que para alcançar um nível desportivo internacional no karaté pode ser importante incrementar os níveis de CMJ até aos valores similares aqui revelados.

Palavras-chave: Artes marciais; desportos de Combate; kata; força explosiva; alto rendimento.

\section{1.- Introducción}

Las características de la competición de kata en karate exigen a los deportistas unos niveles de condición física elevados, en especial en lo relativo a la producción de potencia anaeróbica (Chaabène, Hachana, Franchini, Mkaouer, \& Chamari, 2012). Por ello, las necesidades de producir fuerza a altas velocidades son elevadas pues, entre otros factores, la velocidad de ejecución de los movimientos es un criterio fundamental de puntuación en las competiciones de katas (World Karate Federation, 2013). En concreto, la fuerza explosiva del tren inferior cobra una gran relevancia en los estudios del perfil físico de los karatekas, dada la cantidad de técnicas que exigen patadas veloces y saltos elevados (Busko \& Wit, 2002; Pozo, Bastien, \& Dierick, 2011; ScattoneSilva, Lessi, Lobato, \& Serrão, 2012; VencesBrito, Rodrigues Ferreira, Cortes, Fernandes, \& PezaratCorreia, 2011; Young Kwan, Yoon Hyuk, \& Shin Ja, 2011). Así, en los estudios existentes en la literatura sobre la valoración de la fuerza en karatekas es muy frecuente encontrar análisis de la capacidad de salto vertical y de la velocidad de patada (Chaabène et al., 2012; Ravier, Grappe, \& Rouillon, 2004; Wong et al., 2010). Por una parte, la evaluación del salto vertical constituye uno de los métodos de valoración de la fuerza explosiva de miembros inferiores más usados en multitud de especialidades deportivas dada su capacidad de detectar estados de fatiga y efectos del entrenamiento (Balsalobre-Fernández, Tejero-González, Campo-Vecino, \& Alonso-Curiel, 2013; Gorostiaga et al., 2004; Jiménez-Reyes \& González-Badillo, 2011; Sanchez-Medina \& GonzálezBadillo, 2011). Por otra parte, el análisis biomecánico de las técnicas de patada es una prioridad en los estudios en artes marciales, ya que dichas habilidades tienen una gran presencia tanto en las competiciones de combates como en las de katas (Pozo et al., 2011; Quinzi, Camomilla, Felici, Di Mario, \& Sbriccoli, 2013; Sforza et al., 2002).

Por todo ello, de cara a perfeccionar tanto los métodos de entrenamiento como los procesos de selección del talento deportivo, se torna indispensable conocer qué niveles de salto vertical y velocidad de patada son necesarios para alcanzar el éxito competitivo. Para tal fin, es necesario identificar qué diferencia a karatekas con medallas internacionales de karatekas de nivel nacional y amateur en cuanto a dichas variables se refiere, pues ello permitirá conocer qué características especiales poseen los deportistas de élite. En concreto, sería de gran interés conocer las posibles diferencias en, por un lado, el salto CMJ, al ser probablemente el test de potencia de miembros inferiores más utilizado por su gran validez y fiabilidad (Jiménez-Reyes \& González-Badillo, 2011) y, por otro lado, la velocidad de la patada mae-geri, al ser la técnica de pierna más utilizada en los katas de karate (Pozo et al., 2011; Sforza et al., 2002).

Tratando de abordar esta línea de investigación, Ravier, Grappe y Rouillon (2004), encontraron que los karatekas junior franceses de élite tenían unos valores de CMJ 
significativamente superiores a los de nivel nacional, siendo la diferencia media de $4,9 \mathrm{~cm}$. Cabe destacar que en diversos estudios se han encontrado niveles de salto vertical superiores a los 40 cm. en karatekas de nivel internacional (Doria et al., 2009; Koropanovski et al., 2011; Roshcel et al., 2009), lo cual pone de manifiesto que los karatekas de alto nivel poseen unos valores de salto vertical similares a los de, por ejemplo, atletas de alto nivel de 400 metros vallas, cuyos altos niveles de potencia anaeróbica son conocidos (Balsalobre-Fernández et al., 2013). En cuanto a la velocidad de ejecución de la patada mae-geri, diversos estudios (Pozo et al., 2011; Sforza et al., 2002) han comprobado que los karatekas de mayor nivel competitivo poseen niveles más altos de velocidad que los deportistas menos expertos, aunque hay una gran escasez de estudios al respecto.

En definitiva, los pocos estudios existentes en la bibliografía que analizan las diferencias en cuanto a la fuerza explosiva de miembros inferiores de karatekas de diversos niveles competitivos, parecen concluir que los más expertos tienen valores más altos que los más amateurs. Sin embargo, dichos estudios cuentan con participantes junior o de un menor nivel competitivo, y en ningún caso estudian a karatekas senior de alto éxito internacional. Además, la literatura científica apenas tiene estudios sobre las diferencias en la fuerza explosiva de los miembros inferiores y la velocidad de patada entre karatekas de éxito internacional y practicantes de nivel nacional/amateur, lo cual tiene una gran utilidad práctica para entrenadores y deportistas pues el conocimiento de dichas diferencias podrá permitir, por un lado, optimizar los métodos de entrenamiento de los deportistas amateurs para acercarse a los valores de los deportistas exitosos y, por otro lado, identificar los posibles talentos deportivos mediante la medición de unos test de campo sencillos y poco costosos. Por todo ello, este trabajo tiene el objetivo de comparar las diferencias en el salto vertical CMJ y la velocidad de mae-geri entre karatekas de alto éxito internacional y karatekas de nivel amateur/nacional.

\section{2.- Método}

\section{1.-Participantes}

La muestra estuvo formada por 24 karatecas españoles masculinos, especialidad katas. Se diferenciaron dos submuestras. Una formada por 13 karatecas de alto nivel que han participado de forma exitosa en competición internacional - con un total como colectivo de 77 medallas de oro a bronce en campeonatos senior y junior de Europa y del Mundo-, y edad entre 15 y 27 años, talla entre 157 y $177 \mathrm{~cm}$, peso entre 56.2 y $72.5 \mathrm{~kg}$ e Índice de Masa Corporal (IMC) entre 20.0 y 25.2 $\mathrm{kg} / \mathrm{m}^{2}$. La segunda submuestra se configuró con 11 karatecas que han participado en competiciones amateurs o nacionales del Estado español, pero no en competición internacional, y edad entre los 14 y los 25 años, talla entre 159 y $183.3 \mathrm{~cm}$, peso entre 51.2 y $87.3 \mathrm{~kg}$ e IMC entre 18.6 y $26.6 \mathrm{~kg} / \mathrm{m}^{2}$. La participación de los deportistas fue voluntaria y consentida. Se respetó la Declaración de Helsinki y el estudio fue aprobado y autorizado por la Federación Madrileña de Karate (España). Ver Tabla 1.

Tabla 1. Características de la muestra

\begin{tabular}{|c|c|c|c|c|c|}
\hline $\mathbf{N}$ & Edad (años) & Talla (cm) & Peso (kg) & IMC & Logros Deportivos \\
\hline 13 & $21 \pm 3.4$ & $168.5 \pm 6.7$ & $66.8 \pm 7.3$ & $23.5 \pm 1.7$ & $\begin{array}{l}\text { MEDALLAS (total: 77). Campeonatos del Mundo: } 1 \\
\text { oro, } 4 \text { platas, } 6 \text { bronces. Campeonatos de Europa: } \\
22 \text { oros, } 19 \text { platas, } 25 \text { bronces. }\end{array}$ \\
\hline 11 & $17 \pm 3.5$ & $170.5 \pm 7.6$ & $65.2 \pm 10.8$ & $22.3 \pm 2.6$ & $\begin{array}{l}\text { Participación en competiciones amateurs o } \\
\text { nacionales. }\end{array}$ \\
\hline
\end{tabular}

\section{2.- Diseño y procedimiento}

El estudio siguió un diseño ex post facto retrospectivo. Todos los participantes realizaron el mismo calentamiento, el cual fue dirigido por un maestro en karate, cinturón negro 3er DAN y entrenador nacional de karate. El calentamiento consistió en carrera continua combinado con diez 
flexiones de pierna y diez saltos. Luego realizaron quince flexiones de brazos. Para acabar los deportistas realizaron 20 puñetazos frontales (tsukis) y 20 patadas frontales (mae-geri). Posteriormente, se procedió a la medición de las variables en el siguiente orden: salto vertical CMJ y velocidad de ejecución de la patada mae-geri, siguiendo el orden de menor a mayor carga metabólica como aconseja la NSCA (Coburn \& Malek, 2011). El estudio fue llevado a cabo en las instalaciones deportivas de la Federación Madrileña de Karate.

\section{3.- Salto vertical CMJ}

Para la medición del salto vertical CMJ se utilizó una plataforma de infrarrojos Optojump, cuya fiabilidad y validez ha sido demostrada (Glatthorn et al., 2011). Las manos se situaron en las caderas y el salto se realizó verticalmente y de forma enérgica intentando llegar lo más alto posible, aterrizando en el mismo lugar en el que se despegó. Los deportistas realizaron tres intentos, tomando en consideración la media de los tres saltos. El valor de cada salto se obtuvo en centímetros.

\section{4.- Velocidad de patada mae-geri}

La patada mae-geri se realizó desde la posición anatómica, en bipedestación con los pies separados a la anchura de las caderas y los brazos colocados a lo largo del cuerpo, y se dijo a los karatekas que la ejecutaran a la máxima velocidad posible. Dicha técnica se divide en 3 fases bien descritas en Pozo et al. (2011), y básicamente consiste en un flexión de la cadera en el plano sagital, con la rodilla flexionada, y una posterior extensión de rodilla hacia delante en dicho plano.

Para la medición de la velocidad de la patada mae-geri, se utilizó una cámara de alta velocidad (Casio EXFC-100) con una frecuencia de 240 fotogramas por segundo y una calidad de 448x336 píxeles con la que se grabó cada patada de los karatecas. La cámara se colocó en un trípode situado de manera perpendicular al plano sagital de los deportistas y a una distancia de los mismos de 2,5 m., lo cual permitía grabar a los sujetos de perfil desde los pies hasta la cabeza. Se colocó un marcador en los maléolos externos de cada deportista que servía como marcador de posición y, con ellos, cada deportista realizó 3 mae-geris con cada pierna. Posteriormente, se analizó cada vídeo grabado mediante el software biomecánico de análisis de videos Kinovea 0.8.15 para Windows, con el que se obtuvieron datos de velocidad media, y tiempo de ejecución de cada patada. Para ello, el software tomó como referencia los marcadores de los maléolos externos y se consideró el inicio del movimiento el punto en el que el pie deja de contactar el suelo, y como fin del movimiento el punto en el que la velocidad de desplazamiento del marcador se vuelve cero, en la última fase de extensión del mae-geri. Se ajustó la frecuencia de origen a $240 \mathrm{~Hz}$ y se tomó como referencia una distancia conocida de $1 \mathrm{~m}$ situada en el mismo plano de ejecución de la patada maegeri (en el suelo) para el cálculo de la velocidad de la misma. Hecho esto, se calculó la velocidad media mediante la herramienta de seguimiento automático de trayectoria del Kinovea en cada fotograma del video, y la velocidad media final se calculó como la media de todas ellas. Una vez analizados todos los vídeos, se estimó el promedio de los 6 mae-geri de cada deportista en una variable: velocidad media de ejecución (en $\mathrm{m} / \mathrm{s}$ ). Los datos son altamente fiables: dos analizadores realizaron el análisis de los vídeos de forma independiente y por separado, obteniendo un Coeficiente de Correlación Intraclase de 0.91.

\section{5.- Análisis estadístico}

Se testó la normalidad de las variables mediante el estadístico Shapiro-Wilk. Posteriormente se llevó a cabo un análisis multivariable de la varianza con covariable (MANCOVA); en concreto, se comparó a las dos submuestras (internacionales vs. amateurs/nacionales) en las variables salto vertical y velocidad promedio del mae-geri, controlando el posible efecto que tuviera sobre ellas la covariable años de edad. Para la estimación del tamaño del efecto se utilizó el estadístico eta al cuadrado parcial $\left(\eta^{2}\right)$. El nivel de confianza establecido fue del $95 \%(\mathrm{p}<, 05)$. Los resultados fueron estimados con ayuda del programa informático IBM SPSS Statistics 20. 


\section{3.- Resultados}

El estadístico Shapiro-Wilk informó de que las variables del estudio se distribuyeron normalmente ( $p>0.05$ en todas las ocasiones). Se procedió con técnica MANCOVA, encontrando un modelo multivariante estadísticamente significativo (Intersección: Wilks' Lambda=0.13, F=62.28, $\mathrm{p}<0.001$; Edad: Wilks' Lambda=0.64, $\mathrm{F}=5.40, \mathrm{p}<0.013$; Rendimiento: Wilks' Lambda=0.68, $\mathrm{F}=4.51$, $\mathrm{p}<0.024)$. Así, después de controlar el efecto de la edad sobre el resto de variables, se observó en relación con el salto vertical que los karatecas que competían a nivel internacional saltaban más $(46.4 \pm 4.2 \mathrm{~cm})$ que los que competían de forma aficionada $(38 \pm 4.6 \mathrm{~cm})$, siendo una diferencia estadísticamente significativa $\left(F=9,47 ; p=0,006 ; \eta^{2}=0,311\right)$. Sin embargo, en relación con la variable velocidad media de mae-geri, los resultados obtenidos por los karatecas internacionales $(19.3 \pm 1,4)$ y los aficionados o nacionales $(18.2 \pm 0.96)$ no se diferenciaron estadísticamente $(\mathrm{F}=0.80 ; p=0.38$; $\left.\eta^{2}=0.03\right)$. Ver Tabla 2 y Figura 1.

Tabla 2. Prueba MANCOVA

\begin{tabular}{|c|c|c|c|c|c|c|}
\hline & \multicolumn{2}{|c|}{ Internacionales $(n=13)$} & \multicolumn{2}{|c|}{ Nacionales $(\mathrm{n}=11)$} & \multirow[b]{2}{*}{$\boldsymbol{p}$} & \multirow{2}{*}{$\eta^{2}$} \\
\hline & $\mathbf{M}$ & DT & $\mathbf{M}$ & DT & & \\
\hline Altura en salto vertical CMJ (cm) & 46.4 & 4.2 & 38.0 & 4.67 & 0.006 & 0.311 \\
\hline Promedio velocidad media $(\mathrm{m} / \mathrm{s})$ & 19.3 & 1.49 & 18.2 & 0.96 & 0.38 & 0.03 \\
\hline
\end{tabular}

Abreviaturas: $\mathrm{M}=$ media aritmética, $\mathrm{DT}=$ desviación típica, $\mathrm{p}=$ Probabilidad de significación, prueba $\mathrm{F}$ de MANCOVA, $\eta^{2}=$ tamaño del efecto, eta cuadrada parcial.

Figura 1. Diferencias porcentuales según el nivel deportivo en la altura en CMJ y la velocidad de mae-geri

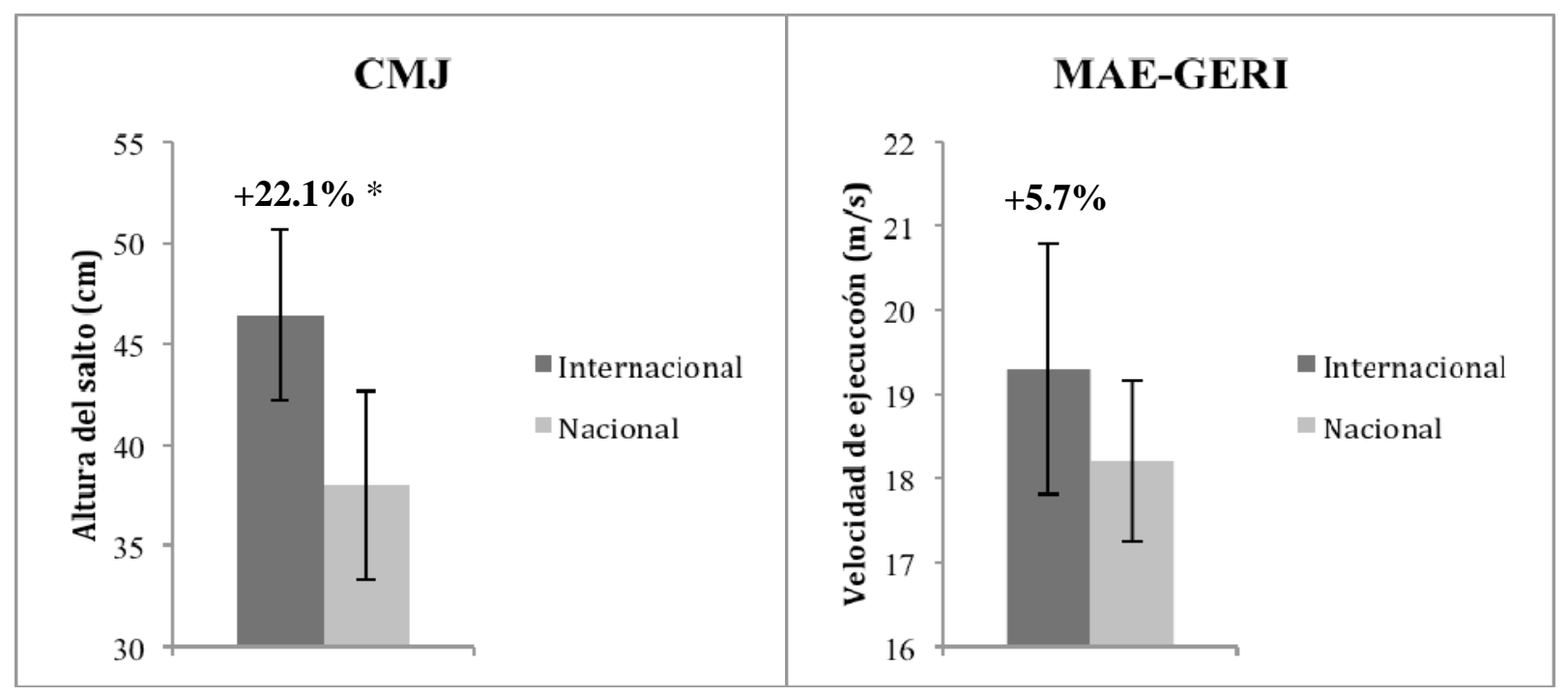

* Diferencias significativas con $\mathrm{p}<0.05$

\section{4.- Discusión y conclusiones}

Los resultados de nuestro estudio confirman la hipótesis de que los karatekas de élite tienen niveles de fuerza explosiva de miembros inferiores significativamente mayores a los de los karatekas de nivel nacional/amateur. Estos resultados concuerdan con los de otros autores que comprobaron que los karatekas de nivel internacional tienen mayores valores de salto vertical CMJ y velocidad de patada que los de menor nivel competitivo (Chaabène et al., 2012; Pozo et al., 2011). Es más, los niveles de salto vertical alcanzados por los participantes de nuestro estudio $46.4 \mathrm{~cm}$ de promedio) son incluso superiores a los de atletas de alto nivel de una especialidad tan explosiva como el 400 metros vallas (Balsalobre-Fernández et al., 2013). En este sentido, nuestro estudio refuerza los hallazgos de los otros pocos estudios que han abordado esta cuestión, y, además, lo hace con una muestra del máximo nivel competitivo en la que se encuentran múltiples Campeones, Subcampeones y Medallas de Bronce de Europa y del Mundo. Parece que queda claro, por tanto, 
que una característica común a los karatekas de alto nivel competitivo es su elevada capacidad de salto vertical.

Este hallazgo tiene dos aplicaciones prácticas de gran relevancia para el desarrollo del karate. Por un lado, sería razonable aconsejar a técnicos y deportistas que desarrollen programas de entrenamiento con cargas que tengan como objetivo la mejora de la fuerza explosiva de los miembros inferiores de los karatekas para aproximarlos a los niveles de los competidores de élite. Los programas de entrenamiento de fuerza con cargas se han utilizado satisfactoriamente en numerosas ocasiones para mejorar la fuerza explosiva de miembros inferiores de muy diversos tipos de deportistas (Balsalobre-Fernández et al., 2013; de Villarreal, Izquierdo, \& Gonzalez-Badillo, 2011; González-Badillo, Gorostiaga, Arellano, \& Izquierdo, 2005; Gorostiaga et al., 2004; Ignjatović, Stanković, Marković, \& Milanović, 2011), y, sin embargo, sólo conocemos algunas pocas reseñas en la literatura científica que muestren la efectividad de dichos entrenamientos en la mejora del rendimiento de los karatekas (Voigt \& Klausen, 1990). No obstante, hasta donde llega nuestro conocimiento, no existen estudios que traten esta cuestión con una muestra de élite y con tecnologías precisas para registrar los cambios en la producción de fuerza como pueden ser los transductores lineales de posición y velocidad (González-Badillo \& Sánchez-Medina, 2010). Por todo ello, creemos que un paso lógico en el horizonte investigador sería analizar la efectividad de uno o varios programas de entrenamiento de fuerza específicamente diseñados para mejorar la capacidad de salto vertical.

Por otro lado, los resultados encontrados en esta investigación pueden ofrecer una valiosa información para la selección del talento deportivo, pues si los karatekas de élite poseen mayores valores de salto que los menos entrenados, sería aconsejable proponer a técnicos y entrenadores la valoración de dicha capacidad para identificar aquellos deportistas principiantes con unos niveles de fuerza explosiva de miembros inferiores lo más cercanos posibles a los de los karatekas de éxito internacional. Así, este sencillo análisis puede ayudar a detectar a aquellos deportistas que, al menos desde el punto de vista físico, tenga unas capacidades más apropiadas para progresar hacia el deporte de alto nivel. No obstante, sería interesante continuar esta línea de investigación para descubrir otras variables comunes de los karatekas de alto nivel competitivo más allá del salto vertical, pues ello ayudará a configurar un perfil físico completo de este tipo de deportistas, lo cual puede aportar información clave para el entrenamiento de estos atletas.

Sin embargo, el MANCOVA no mostró diferencias en la velocidad de mae-geri entre los dos grupos de karatekas cuando se controla la edad. Esto quiere decir que el hecho de que los karatekas internacionales tengan mayor velocidad en la patada mae-geri se debe a que tienen mayor edad y, con ello, más años de entrenamiento. No obstante, estos resultados deben interpretarse con cautela dado el reducido tamaño muestral del estudio. Esta diferencia de edad es la principal limitación del estudio, pues podría argumentarse que los karatekas de menor nivel tienen menor nivel precisamente por ser más jóvenes. Sin embargo, el grupo de karatekas internacionales ya tenían un abultado palmarés en competiciones junior y cadete de carácter internacional con la misma edad que actualmente tienen los participantes pertenecientes al grupo nacional/amateur (Europeos y/o Mundiales), mientras que los sujetos del grupo nacional/amateur sólo han competido como mucho en Campeonatos de España y sin obtener medalla. En cualquier caso, reconociendo la limitación del presente estudio, son necesarias más investigaciones con muestras más amplias y edades homogéneas para saber si la velocidad en la patada mae-geri diferencia o no a karatekas internacionales de los de nivel amateur/nacional cuando se controla la edad mediante MANCOVA.

Por su parte, la altura alcanzada en el salto vertical CMJ, aun habiendo controlado el factor edad para el análisis de la diferencia de medias inter-grupos, sigue siendo significativamente mayor en los karatekas internacionales que en los de nivel amateur/nacional. Así, la altura alcanzada en el salto vertical CMJ ha mostrado ser una variable independiente de los años de edad y capaz de diferenciar a karatekas de éxito de los de menor nivel de rendimiento. De esta forma, este hallazgo no hace sino reafirmar los estudios que afirman que el salto vertical CMJ es una prueba con una gran capacidad de detectar el nivel de rendimiento físico de los deportistas de diferentes disciplinas (Balsalobre-Fernández et al., 2013; Jiménez-Reyes \& González-Badillo, 2011; Sanchez-Medina \& González-Badillo, 2011). Además, el salto vertical CMJ tiene la ventaja de ser prácticamente inocuo al deportista, de tal forma que su valoración no supondrá ninguna alteración en su carga de 
entrenamiento, como si lo pueden hacer otras valoraciones de la fuerza como, por ejemplo, un test de Repetición Máxima (González-Badillo \& Sánchez-Medina, 2010). De hecho, la evaluación del CMJ puede realizarse semanalmente durante meses sin interferir en el desarrollo de los programas de entrenamiento de los atletas (Jiménez-Reyes \& González-Badillo, 2011).

En conclusión, la notable altura alcanzada en el salto vertical CMJ es una característica común a los karatekas de élite, independiente de la edad y de fácil aplicación práctica, por lo que su valoración puede adquirir una importante relevancia en el proceso de entrenamiento de los karatekas. Esta es la primera investigación sobre las diferencias en la fuerza explosiva de miembro inferior con karatekas españoles de máximo nivel competitivo.

\section{Referencias}

Balsalobre-Fernández, C., Tejero-González, C. M., Campo-Vecino, J. d., \& Alonso-Curiel, D. (2013). The Effects of a Maximal Power Training Cycle on the Strength, Maximum Power, Vertical Jump Height and Acceleration of High-Level 400-Meter Hurdlers. Journal of Human Kinetics, $36,119-126$.

Busko, K., \& Wit, B. (2002). Force-velocity relationship of lower extremity muscles of karate athletes and rowers. Biology of Sport, 19(4), 373-384.

Chaabène, H., Hachana, Y., Franchini, E., Mkaouer, B., \& Chamari, K. (2012). Physical and Physiological Profile of Elite Karate Athletes. Sports Medicine, 42(10), 829-843.

Coburn, J. W., \& Malek, M. H. (2011). NSCA's Essentials of Personal Training (2nd ed.): Human Kinetics Publishers.

de Villarreal, E. S. S., Izquierdo, M., \& Gonzalez-Badillo, J. (2011). Enhancing jump performance after combined vs. maximal power, heavy-resistance, and plyometric training alone. Journal of Strength and Conditioning Research, 25(12), 3274-3281.

Doria, C., Veicsteinas, A., Limonta, E., Maggioni, M. A., Aschieri, P., Eusebi, F., . . Pietrangelo, T. (2009). Energetics of karate ( kata and kumite techniques) in top-level athletes. European Journal Of Applied Physiology, 107(5), 603-610.

Glatthorn, J. F., Gouge, S., Nussbaumer, S., Stauffacher, S., Impellizzeri, F. M., \& Maffiuletti, N. A. (2011). Validity and reliability of Optojump photoelectric cells for estimating vertical jump height. Journal of Strength \& Conditioning Research, 25(2), 556-560.

González-Badillo, J., Gorostiaga, E. M., Arellano, R., \& Izquierdo, M. (2005). Moderate resistance training volume produces more favorable strength gains than high or low volumes during a short-term training cycle. Journal of Strength \& Conditioning Research, 19(3), 689-697.

González-Badillo, J., \& Sánchez-Medina, L. (2010). Movement Velocity as a Measure of Loading Intensity in Resistance Training. International Journal of Sports Medicine, 31(5), 347-352.

Gorostiaga, E. M., Izquierdo, M., Ruesta, M., Iribarren, J., González-Badillo, J. J., \& Ibáñez, J. (2004). Strength training effects on physical performance and serum hormones in young soccer players. European Journal of Applied Physiology, 91(5-6), 698-707.

Ignjatović, A., Stanković, R., Marković, Ž., \& Milanović, S. (2011). Effects of resistance training program on dynamic muscle potential in young basketball players. Research in Kinesiology, 39(1), 37-42.

Jiménez-Reyes, P., \& González-Badillo, J. J. (2011). Monitoring training load through the CMJ in sprints and jump events for optimizing performance in athletics. Cultura, Ciencia y Deporte, 7(18), 207-217.

Koropanovski, N., Berjan, B., Bozic, P. R., Pazin, N., Sanader, A., Jovanovic, S., \& Jaric, S. (2011). Anthropometric and Physical Performance Profiles of Elite Karate Kumite and Kata Competitors. Journal of Human Kinetics, 30, 107-114.

Pozo, J., Bastien, G., \& Dierick, F. (2011). Execution time, kinetics, and kinematics of the mae-geri kick: Comparison of national and international standard karate athletes. Journal of Sports Sciences, 29(14), 1553-1561.

Quinzi, F., Camomilla, V., Felici, F., Di Mario, A., \& Sbriccoli, P. (2013). Differences in neuromuscular control between impact and no impact roundhouse kick in athletes of different skill levels. Journal of Electromyography \& Kinesiology, 23(1), 140-150. 
Ravier, G., Grappe, F., \& Rouillon, J. D. (2004). Application of force-velocity cycle ergometer test and vertical jump tests in the functional assessment of karate competitor. Journal of Sports Medicine \& Physical Fitness, 44(4), 349-355.

Roshcel, H., Batista, M., Monteiro, R., Bertuzzi, R., Barroso, R., Loturco, I., . . Franchini, E. (2009). Association between neuromuscular tests and kumite performance on the Brazilian Karate National Team. Journal of Sports Science \& Medicine, 8(3), 20-24.

Sanchez-Medina, L., \& González-Badillo, J. J. (2011). Velocity Loss as an Indicator of Neuromuscular Fatigue during Resistance Training. Medicine \& Science in Sports \& Exercise, 43(9), 1725-1734.

Scattone-Silva, R., Lessi, G. C., Lobato, D. F. M., \& Serrão, F. V. (2012). Acceleration time, peak torque and time to peak torque in elite karate athletes. Science \& Sports, 27(4), e31-e37.

Sforza, C., Turci, M., Grassi, G. P., Shirai, Y. F., Pizzini, G., \& Ferrario, V. F. (2002). Repeatability of mae-geri-keage in traditional karate: a three-dimensional analysis with black-belt karateka. Perceptual \& Motor Skills, 95(2), 433-444.

VencesBrito, A. M., Rodrigues Ferreira, M. A., Cortes, N., Fernandes, O., \& Pezarat-Correia, P. (2011). Kinematic and electromyographic analyses of a karate punch. Journal of Electromyography \& Kinesiology, 21(6), 1023-1029.

Voigt, M., \& Klausen, K. (1990). Changes in muscle strength and speed of an unloaded movement after various training programmes. European Journal of Applied Physiology \& Occupational Physiology, 60(5), 370-376.

Wong, D. P., Tan, E. C. H., Chaouachi, A., Carling, C., Castagna, C., Bloomfield, J., \& Behm, D. G. (2010). Using squat testing to predict training loads for lower-body exercises in elite karate athletes. Journal of Strength \& Conditioning Research, 24(11), 3075-3080.

World-Karate-Federation. Competition Rules. Disponible en http://www.wkf.net/images/downloads/WKF COMPETITION RULES VERSION 80 esp.pdf. Recuperado el 22 de Junio de 2013.

Young Kwan, K., Yoon Hyuk, K., \& Shin Ja, I. (2011). Inter-joint coordination in producing kicking velocity of Taekwondo kicks. Journal of Sports Science \& Medicine, 10(1), 31-38.

\section{Datos biográficos de los autores}

Carlos Balsalobre-Fernández (España) es Personal Investigador en Formación (Beca FPI), Universidad Autónoma de Madrid, 2012-2016. Licenciado en Ciencias de la Actividad Física y del Deporte, Universidad Autónoma de Madrid 2007-2011. Premio a mejor expediente académico. Máster Oficial en Rendimiento Físico y Deportivo, Universidad Pablo de Olavide, 2011-2012. Máster en Alto Rendimiento Deportivo, Comité Olímpico Español-Universidad Autónoma de Madrid, 2011-2013. Especialista en Fuerza y Preparación física por la National Strength \& Conditioning Association (CSCS-NSCA). Cinturón negro de Karate y cinturón negro de Wushu. E-mail: carlos.balsalobre@uam.es

Víctor Martínez-Majolero (España) es Máster en Alto Rendimiento Deportivo Comité olímpico EspañolUniversidad Autónoma de Madrid, 2010-2012. Licenciado en Ciencias de la Actividad Física y el Deporte, Universidad Autónoma de Madrid, 2005-2009. Deportista de alto nivel reconocimiento publicado por el BOE el 11/12/2008. Miembro del equipo nacional de karate, siendo Subcampeón del Mundo Universitario. Tercero del Mundo Universitario. Tercero del Mundo. Dos veces Campeón de Europa. Tercero de Europa. Profesor de Karate. Cinturón Negro 3er DAN por la Real Federación Española de Karate. Entrenador Nacional de Karate por la Real Federación Española de Karate. E-mail: victorm.majolero@gmail.com

Jorge Villacieros-Rodríguez (España) es Coordinador de la Escuela Madrileña de Preparadores de la Federación Madrileña de Karate. Licenciado en Ciencias de la Actividad Física y del Deporte, Universidad Autónoma de Madrid 2005-2009. Máster Oficial en Actividad Física y Salud, Universidad Europea de Madrid, 2009-2011. Entrenador Regional de Karate, Federación Madrileña de Karate, 2013. Cinturón Negro 2o DAN de Karate. E-mail: jvillrod@uax.es

Carlos Ma Tejero-González (España) es Profesor Contratado Doctor de la Universidad Autónoma de Madrid. Profesor del Máster en Alto Rendimiento Deportivo del Comité Olímpico de Estudios Superiores. Doctor en Métodos de Investigación. Máster en Estadística Aplicada. Máster en Psicología del Deporte. Licenciado en Psicología. Licenciado en Ciencias del Deporte. E-mail: carlos.tejero@uam.es. 